\title{
Insuficiência valvar aórtica por valva quadricúspide
}

\author{
Marco Antônio SALUM*, Karina Santos WANDECK ${ }^{* *}$, Márcio KALIL***, \\ Renato N. F. OLIVEIRA*, Gilberto Lino VIEIRA*
}

RBCCV 44205-577

\begin{abstract}
Salum M A, Wandeck K S, Kalil M, Oliveira R N F, Viera G L - Insuficiência valvar aórtica por valva quadricúspide. Rev Bras Cir Cardiovasc 2002; 17(1): 99-101.

RESUMO: Valva aórtica quadricúspide (VAQ) é uma anomalia rara com incidência entre 0,003\% e $0,043 \%$. Pode ser achado ocasional em pacientes assintomáticos, mas, freqüentemente, está associada com regurgitação aórtica. O ecocardiograma é método ideal de determinação e acompanhamento dessa anomalia e suas repercussões. É relatado caso de paciente do sexo masculino, 56 anos, hipertenso, que apresentou dispnéia súbita, tosse seca, sopro diastólico grau III/VI focos aórtico e aórtico acessório, crepitações pulmonares nas bases, sugerindo insuficiência cardíaca. Ecocardiograma transtorácico mostrou VAQ com regurgitação moderada. Houve melhora com tratamento clínico. Após um ano, novo ecocardiograma revelou regurgitação aórtica importante, hipertensão pulmonar, hipertrofia moderada e dilatação leve de ventrículo esquerdo. Diante da deterioração da fração de ejeção e demais achados ecocardiográficos, indicou-se a troca valvar. A valva nativa apresentava três cúspides de mesmo tamanho e uma pouco menor. O paciente evoluiu com remissão dos sintomas. Insuficiência aórtica por valva quadricúspide geralmente é detectada em adultos; nas crianças a função cardíaca é normal. Defeitos cardíacos associados são raros e incluem defeitos óstio coronário. A anatomia valvar varia com o tamanho das cúspides, sendo que em $60 \%$ dos casos a VAQ possui três cúspides de igual tamanho e uma cúspide menor. A definição ecocardiográfica característica de $V A Q$ é a forma de ' $X$ ' durante a diástole e abertura livre das cúspides com configuração retangular durante a sístole. Os achados clínicos e anatômicos da valva do paciente descrito condizem com a literatura.
\end{abstract}

DESCRITORES: Valva aórtica, cirurgia. Insuficiência da valva aórtica, cirurgia. Valva aórtica, patologia.

\section{INTRODUÇÃO}

A valva aórtica quadricúspide (VAQ) é uma anomalia rara, cuja incidência estimada encontra-se entre $0,003 \%$ e $0,043 \%{ }^{(1-5)}$. Pode ser um achado ocasional em pacientes assintomáticos, mas, freqüentemente, está associada com regurgitação aórtica (1-3). Geralmente, não está associada a outros defeitos cardíacos. O ecocardiograma é o método ideal de determinação e acompanhamento desta anomalia e suas repercussões (1-5). O objetivo deste trabalho é relatar um caso desta anomalia.

\section{RELATO DE CASO \\ Paciente de 56 anos do sexo masculino, leucodérmico, em controle de hipertensão arterial sistêmica com níveis pressóricos mantidos em 140/}

Trabalho realizado no Hospital Mater Dei. Belo Horizonte, MG, Brasil. Recebido para publicação em setembro de 2001.

* Do Serviço de Cirurgia Cardiovascular do Hospital Mater Dei. Belo Horizonte

**Acadêmica da Faculdade de Medicina da Universidade Federal de Minas Gerais.

*** Da Fundação BDMG de Seguridade Social - DESBAN.

Endereço para correspondência: Marco Antônio Salum. Av. Brasil 691, sala 202. Belo Horizonte, MG, Brasil. CEP: 30140-000. Tel. (31) 3273-2277. e.mail: salum@cardiol.br 
$70 \mathrm{mmHg}$, em uso de diurético de alça e inibidor do receptor da angiotensina II. Tinha evolução satisfatória quando apresentou dispnéia paroxística noturna e tosse seca, que o fizeram procurar assistência médica. Ao exame físico, encontrava-se sem edemas, com pulsos rítmicos, simétricos, em "martelo d'água", precórdio hiperdinâmico, ictus impulsivo e sopro diastólico grau III/VI em focos aórtico e aórtico acessório, bem como crepitações pulmonares em ambas as bases. Com base nesses dados, diagnosticou-se insuficiência cardíaca. A radiografia de tórax apresentava aumento da área cardíaca às custas de câmaras esquerdas. O eletrocardiograma foi compatível com sobrecarga de ventrículo esquerdo. O ecocardiograma transtorácico mostrou valva aórtica com quatro cúspides e regurgitação moderada. Acrescentou-se ao esquema terapêutico a Digoxina, sendo o paciente acompanhado em regime ambulatorial, com melhora do quadro. $O$ paciente fazia uso descontínuo da medicação. Após cerca de um ano, novo ecocardiograma para controle revelou regurgitação aórtica acentuada, dilatação leve da porção ascendente da artéria aorta, hipertrofia moderada e dilatação discreta de ventrículo esquerdo, aumento discreto de câmaras direitas e hipertensão pulmonar. A seguir, foi realizado cateterismo cardíaco que evidenciou a VAQ com insuficiência aórtica grave, contratilidade preservada de ventrículo esquerdo, coronárias isentas de aterosclerose significativa e normoposicionadas. Diante do início da deterioração da fração de ejeção do paciente, da presença de dilatação do ventrículo esquerdo e hipertensão pulmonar, indicou-se a troca valvar (valva biológica, por escolha do paciente). A valva nativa era formada por três cúspides de mesmo tamanho e uma cúspide pouco menor. O paciente evoluiu com hipotensão arterial no pósoperatório imediato, com hemiparesia esquerda transitória (pequenas áreas de isquemia cerebral à tomografia computadorizada), com boa evolução com auxílio de fisioterapia.

\section{COMENTÁRIOS}

A valva aórtica quadricúspide é uma anomalia rara, cuja incidência oscila entre $0,003 \%$ a $0,043 \%$, em contraste com a valva aórtica bicúspide que tem sua incidência estimada em $2 \%$ da população geral ${ }^{(3,4)}$. A insuficiência valvar por VAQ geralmente só é detectada em adultos; nas crianças a função cardíaca é normal ${ }^{(1-4)}$. Os achados anatomopatológicos têm demonstrado que o espessamento fibroso da valva pode resultar em incompleta coaptação das cúspides e conseqüente regurgitação. Outro possível mecanis- mo responsável pela insuficiência valvar consiste na presença de uma cúspide acessória menor, que causaria distribuição desigual do estresse transvalvar e coaptação anormal das cúspides, com resultante trauma localizado e deterioração hemodinâmica ${ }^{(3)}$.

JANSSENS et al. (5), em revisão da literatura, identificaram 70 casos de VAQ, sendo 26 casos diagnosticados pelo ecocardiograma transtorácico ou transesofágico, 25 por necrópsia, 15 durante cirurgias e $4 \mathrm{com}$ angiografia.

Há poucos relatos na literatura sobre defeitos cardíacos associados à $\mathrm{VAQ}$, sendo as anomalias do óstio coronário e artérias coronárias as mais $\operatorname{descritas}^{(4,5)}$.

A anatomia da valva varia de acordo com o tamanho das cúspides, sendo que em $60 \%$ dos casos a $V A Q$ possui três cúspides de igual tamanho e uma cúspide menor (1-5). Em apenas $12 \%$ dos casos, as cúspides são de igual tamanho.

O ecocardiograma é o método de escolha para o diagnóstico precoce e acompanhamento de sua principal complicação, a regurgitação aórtica (1-5). A definição ecocardiográfica característica de $V A Q$ é a forma de ' $X$ ' durante a diástole e abertura livre das cúspides com configuração retangular durante a sístole ${ }^{(1,3)}$.

No paciente deste relato, os sintomas manifestaram-se a partir dos 56 anos de idade, fato condizente com a história natural da anomalia descrita na literatura. Não havia antecedente de febre reumática. Ao ecocardiograma, a valva aórtica era constituída de três cúspides de igual tamanho e uma pouco menor, com imagem em forma de " $X$ " na diástole (Figura 1) e abertura em formato retangular durante a sístole; todos estes dados condizem com outros relatos da literatura.

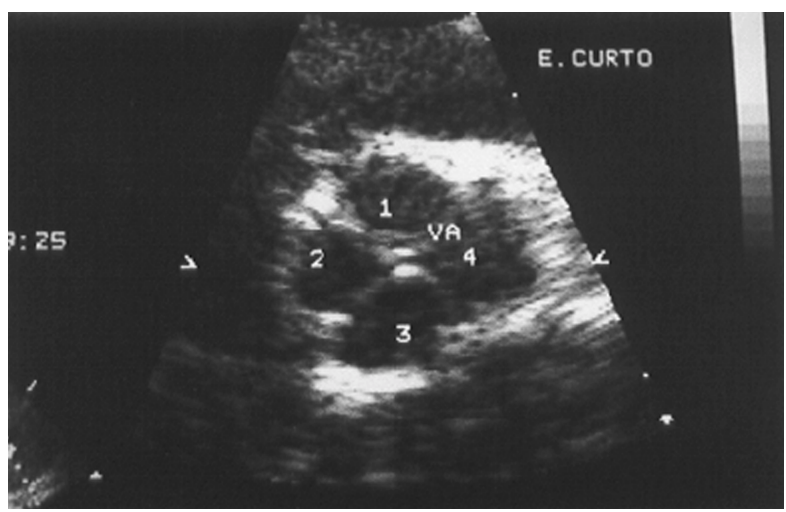

Fig. 1 - Ecocardiograma transtorácico: valva aórtica quadricúspide. 


\section{CONCLUSÕES}

A decisão quanto ao momento cirúrgico ideal tem sido tema controverso. Contudo, parece sensato indicá-la tão logo existam indícios de deterioração da função cardíaca, antes que ocorra dilatação cardíaca.
Embora rara e pouco descrita na literatura, a VAQ é causa de insuficiência cardíaca progressiva e potencialmente refratária ao tratamento medicamentoso, devendo ser incluída no diagnóstico diferencial desses quadros. O ecocardiograma é um exame acessível, não invasivo e que permite o pronto diagnóstico e acompanhamento da anomalia.

RBCCV 44205-577

Salum M A, Wandeck K S, Kalil M, Oliveira R N F, Viera G L - Quadricuspid aortic valve with aortic regurgitation. Rev Bras Cir Cardiovasc 2002; 17(1): 99-101.

ABSTRACT: This article is a case report of a 56 years old man, without known cardiac abnormality, presented signs of cardiac insufficiency. Transthoracic echocardiography revealed a quadricuspid aortic valve (QAV) with a moderated regurgitation. The clinical treatment was initiated with a good evolution. After one year, a new echocardiography revealed huge aortic regurgitation, pulmonary hypertension and lower ejection fraction. After that, the surgery for changing the valve was indicated in order to prevent the pathology progression. QAV is a rare pathology $(0.003 \%$ to $0.043 \%)$. Usually is not associated with other cardiovascular pathologies. It can be an incidental finding but in general it carries aortic regurgitation in adulthood due to bad closure of the four cusps. There are different anatomical variations for the aortic valve cusps: four unequal cusps, three equal cusps and one smaller cusp (majority) and up to four equal cusps. Transthoracic and transoesophageal echocardiography can easily asses the morphological and functional status of such a valve.

DESCRIPTORS: Aortic valve, surgery. Aortic valve, insuffiency. Aortic valve, pathology.

AGRADECIMENTOS: Agradecemos de maneira especial ao Dr. José Gilberto de Brito Henriques, pela ajuda fundamental na elaboração deste trabaIho. Agradecemos, também, ao Dr. Mário Lopez, Dra. Sandra Castilho e Dra. Zilda Alves Meira.

\section{REFERÊNCIAS BIBLIOGRÁFICAS}

1 Feldman B J, Khandheria B K, Warnes C A, Seward J B, Taylor C L, Tajik A J - Incidence, description and functional assessment of isolated quadricuspid aortic valves. Am J Cardiol 1990; 65: 937-8.
2 Brouwer $\mathrm{M} \mathrm{H}$, de Graaf $\mathrm{J} \mathrm{J}$, Ebels $\mathrm{T}$ - Congenital quadricuspid aortic valve. Int J Cardiol 1993; 38:196-8.

3 Cruz L A, Castier M B, Ferro M E, Menezes C C, Albanesi Filho F M - Insuficiência aórtica por valva aórtica quadricúspide. Arq Bras Cardiol 1993; 60:103-5.

4 Prates P R, Lucchese F A, Kalil R A K, Azambuja P, Bertoletti $\mathrm{V}$, Nesralla I A - Valva aórtica quadricúspide com malposição do óstio coronário direito. Arq Bras Cardiol 1977; 30: 369-71.

5 Janssens U, Klues H G, Hanrath P - Congenital quadricuspid aortic valve anomaly associated with hypertrophic nonobstructive cardiomyopathy: a case report and review of the literature. Heart 1997; 78: 83-7. 